\section{Efecto del injerto óseo humano liofilizado y membrana amniótica humana liofilizada en la regeneración ósea en tibias de conejos} Effect of human bone lyophilized graft and human lyophilized amniotic mem-
brane on bone regeneration of rabbit tibias

\section{Resumen}

El presente estudio tuvo como objetivo investigar el efecto del injerto óseo humano liofilizado particulado en combinación con la membrana amniótica humana liofilizada en la regeneración ósea en tibias de conejos. Metodología. Se utilizaron 10 conejos raza Nueva Zelanda, de 6 a 9 meses de edad aproximadamente, hembras y machos en buen estado, de $3.5 \mathrm{~kg}-4 \mathrm{~kg}$ de peso, en los que se crearon defectos circulares óseos de $4 \mathrm{~mm}$ de diámetro en la región más inferior de las extremidades posteriores del conejo (tibias). Se formaron 2 grupos en la tibia de cada conejo: un grupo control (se colocó membrana) y el grupo de estudio (se colocó hueso humano liofilizado y membrana amniótica liofilizada como barrera). Los animales fueron sacrificados a las 16 semanas de la cirugía, y se realizó un análisis clínico e histológico de la zona donde se crearon los defectos óseos. Se concluyó que la regeneración ósea fue significativamente mayor en los defectos cubiertos con hueso humano liofilizado en combinación con membrana amniótica, comparada con las muestras del grupo control, en el que se colocó solo membrana amniótica como barrera.

Palabras clave: Injerto óseo humano liofilizado, membrana amniótica, regeneración ósea.

\begin{abstract}
:
This study aimed to investigate the effect of lyophilized human bone graft in combination with lyophilized human amniotic membrane in bone regeneration of rabbit tibias. Methodology: It were used 10 New Zealand male and female white rabbits 6 to 9 months of age, in good condition, weighing $3.5 \mathrm{~kg}-4$ $\mathrm{kg}$. Two circular bone defects $4 \mathrm{~mm}$ diameter were created in the lower región of the tibias. One of the defects made belonged to control group and was only covered with amniotic membrane, the other defect belonged to the experimental group and was covered with lyophilized human graft plus lyophilized amniotic membrane as a barrier. The animals were sacrificed at 16 weeks after surgery and a clinical and histological analysis of the area of bone defects was done. It was concluded that bone regeneration was significantly higher in the defects covered with lyophilized human bone in combination with amniotic membrane than the control group which took only amniotic membrane as a barrier.
\end{abstract}

Keywords: Human bone graft lyophilized; amniotic membrane; bone regeneration.

Artículo Original

Frank Paredes Nomberto ${ }^{1}$

1. Unidad de Posgrado de la Facultad de Odontología de la Universidad Nacional Mayor de San Marcos, Perú.

Correspondencia:

C.D. Frank Paredes Nomberto

Mz Clote 2 San Diego, Lima 31, Perú.

Correo electrónico: frankpa_no@hotmail.com

\section{Introducción}

La regeneración ósea guiada (ROG) se fundamenta en la utilización de sistemas de barrera mediante membranas que aíslan un determinado defecto óseo, con la intención de evitar el crecimiento de tejidos con capacidad de reparación rápida, como el tejido conectivo, que interfieren con el potencial osteogénico del defecto ${ }^{1} \mathrm{y}$, dependiendo de su acción sobre el hueso se les atribuye capacidad osteogénica, osteoinductiva u osteoconductora ${ }^{2}$.

La RTG tuvo resultados positivos que aprobaron la técnica cuando fue estu- diada en animales. Los estudios clínicos en humanos confirmaron los resultados obtenidos en animales y su uso en periodoncia pasó a ser rutina en aquellos casos en los cuales estaba indicada la técnica. El éxito de esta técnica, basada en la formación de nuevo ligamento periodontal y lógicamente de cemento y hueso, despertó la atención de los investigadores interesados en conseguir un aumento de hueso en otras situaciones clínicas ${ }^{3}$.

Es así que nació la regeneración ósea guiada (ROG). Esta se fundamenta en la utilización de sistemas de barrera me- diante membranas que aíslan un determinado defecto óseo con la intención de evitar el crecimiento de tejidos con capacidad de reparación rápida, como el tejido conectivo, que interfieren con el potencial osteogénico del defecto ${ }^{4,5}$. La membrana utilizada en nuestro estudio fue una lámina de amnios liofilizada, probada por Yabar y col. ${ }^{6}$ en el proceso de regeneración ósea, y el hueso humano liofilizado fue proporcionado por el Instituto Peruano de Energía Nuclear (IPEN). La membrana amniótica es una membrana resistente, transparente, delgada y rica en colágeno que reviste la lámina coriónica y la placenta 
en el período del desarrollo fetal, muy similar a la piel. La membrana amniótica no tiene conductos sanguíneos, conexiones nerviosas ni canales linfáticos.

El grosor del amnios puede variar de 0.02 a $0.4 \mathrm{~mm}$ y consta de cinco capas: epitelio, membrana basal, capa compacta, capa fibroblástica y capa esponjosa. Esta "membrana amniótica liofilizada" hasta la actualidad ha sido utilizada por los especialistas de cirugía plástica y de oftalmología, en el tratamiento de pacientes quemados y reconstrucciones de córnea respectivamente, así como por Yabar y col. in vitro para ver el efecto en el proceso de regeneración ósea. El hueso humano liofilizado para que pueda ser aplicado en la práctica médica requiere estar estéril y la radiación gamma, proveniente del Cobalto-60, es un método eficaz para esterilizar productos para uso biomédico.

Mediante este trabajo de investigación tratamos de introducir el uso de hueso humano liofilizado en combinación con membrana amniótica liofilizada como barrera en el proceso de regeneración ósea guiada. El objetivo general del presente trabajo fue determinar el efecto y comparar el uso del injerto óseo humano liofilizado particulado en combinación con la membrana amniótica humana liofilizada y solamente el uso de membrana amniótica en la regeneración ósea en tibias de conejos.

\section{Materiales y método}

Se utilizaron 10 conejos de raza Nueva Zelanda, de 6 a 9 meses de edad aproximadamente, hembras y machos en buen estado de $3.5-4 \mathrm{~kg}$. de peso. Los animales de experimentación fueron enjaulados en grupos de 5 animales de manera tradicional, se formaron 2 grupos en la misma tibia del conejo, el primero fue el grupo control y el segundo el grupo experimental, los conejos fueron alimentados con comida más agua ad libidum. Después del período de aclimatación, los animales fueron preparados para la implantación quirúrgica del material en estudio. Se retiró el agua y la comida 12 horas antes de la intervención. Se procedió a realizar la depilación de la zona de trabajo (región tibial). La selección, manejo, preparación de los animales y el protocolo quirúrgico se basaron en los principios éticos internacionales del Consejo Internacional de Organizaciones médicas (CIOMS) para investigación biomédica en animales, adoptados en Talloires (1979).
La inducción anestésica se realizó por vía intramuscular (IM), con la administración de clorhidrato de ketamina (35 a $50 \mathrm{mg} / \mathrm{kg}$ ) más clorhidrato de xilacina (5 a $10 \mathrm{mg} / \mathrm{kg}$ ), el nivel de anestesia fue controlado con ketamina IM. El fallecimiento y el mantenimiento de la anestesia fueron controlados mediante la pérdida del reflejo pupilar del ojo, ausencia o presencia de movimientos respiratorios y la relajación del músculo esquelético de la zona quirúrgica. Después de la anestesia, el sitio quirúrgico trabajado fue la región más baja de las extremidades posteriores (tibias). Se procedió a colocar anestesia local (Lidocaína 2\%) y se realizó una incisión de $7 \mathrm{~cm}$ en la superficie media de la tibia extendiéndonos hasta el periostio; luego se hizo el levantamiento del colgajo dejando el hueso expuesto. Se utilizó un sistema de fresas para la preparación de cavidades para implantes cilíndricos de $4 \mathrm{~mm}$ de diámetro con una profundidad de $4 \mathrm{~mm}$ aprox. (Fig. 1), se procedió a colocar el material de investigación (membrana amniótica liofilizada en una cavidad y en la otra membrana amniótica liofilizada en combinación con hueso humano mineralizado liofilizado) para cubrir la entrada de los defectos (membranas de $1 \mathrm{~cm} 2$ ) (Fig. 2).

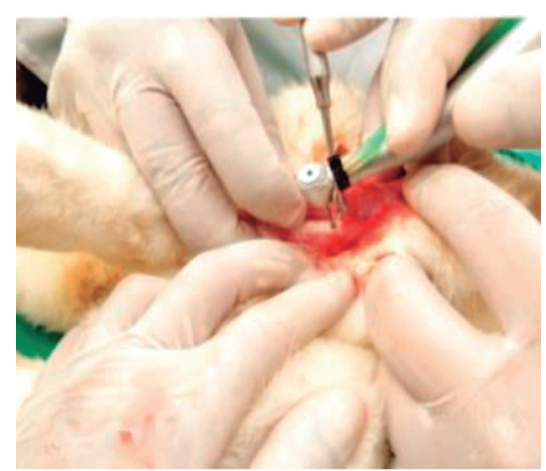

Fig. 1. Preparación de los lechos receptores con fresas.

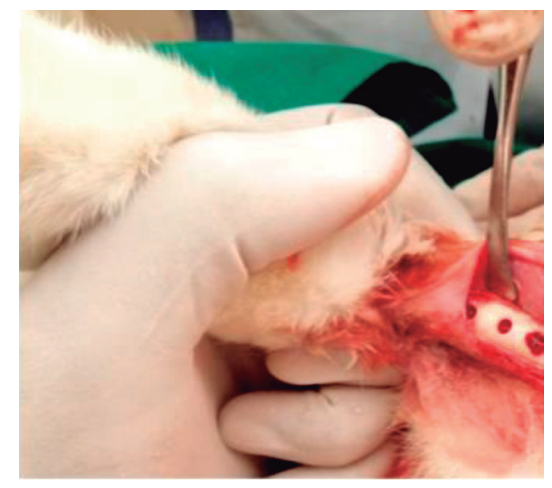

Fig. 2. Grupos control y experimental ensanchadoras de $4 \mathrm{~mm}$ de diámetro y $4 \mathrm{~mm}$ de profundidad.
La membrana y el hueso utilizado en nuestro estudio fueron liofilizados y esterilizados con rayos gamma, los cuales fueron donados por el Banco de Tejidos del Instituto Nacional de Salud del Niño (INSS), institución encargada de la producción de este biomaterial con la ayuda del Instituto Peruano de Energía Nuclear (IPEN). En cada tibia tuvimos 2 sitios de osteotomía. El primer sitio en cada tibia fue el control, que contenía solamente coágulo sanguíneo; el sitio restante fue rellenado con el material de experimentación (hueso humano), ambos grupos fueron cubiertos por la misma membrana amniótica. Se procedió a suturar todos los planos, los más profundos con hilo reabsorbibles (catgut crómico 5 ceros) y los más superficiales con seda negra 3 ceros (4).

Se esperó un período de 16 semanas de cicatrización. Pasadas las 16 semanas, se procedió a sacrificar a los conejos con una dosis de $60 \mathrm{mg} / \mathrm{kg}$ de pentobarbital sódico ${ }^{6}$.

Se determinó el efecto de esta combinación de membrana amniótica y hueso humano liofilizado realizando la valoración en el proceso de regeneración ósea guiada en dos niveles: a nivel clínico (realizando la valoración macroscópica de la zona donde se colocó la membrana después de 16 semanas de cicatrización) y a nivel histológico (mediante las observaciones en microscopia óptica de las muestras obtenidas del proceso de regeneración ósea después de 16 semanas de cicatrización), para lo cual se tomaron en cuenta 2 criterios: la continuidad del defecto óseo - hueso normal y el grado de madurez ósea, el cual esta dado por la presencia de células indiferenciadas, hueso inmaduro o hueso maduro. Con este fin se obtuvieron en total 20 muestras (10 del grupo de estudio y $10 \mathrm{del}$ grupo control), las cuales fueron analizadas clínica e histológicamente.

En la reentrada clínica se observó el grado de inflamación. Para el análisis histológico las muestras de las tibias fueron fijadas en formol al 10\% durante $24 \mathrm{~h}$.

Posteriormente fueron sumergidas en ácido clorhídrico al 10\% durante 2 semanas y luego en ácido nítrico al 10\% durante 1 semana, para completar el proceso de descalcificación. Después se realizaron cortes y se tińeron con la técnica de Hematoxilina- Eosina (H-E). Por último, se observaron al microscopio óptico para determinar histomorfometricamente el proceso de regeneración ósea guiada ${ }^{6}$. 


\section{Resultados}

De un total de 20 muestras, tanto las del grupo de estudio como las del grupo control, se observó clínicamente en todas la ausencia de reacción inflamatoria (100\%), así como ausencia de procesos infecciosos (100\%).

Según el grado de madurez ósea, se encontró hueso maduro compacto en el $100 \%$ en el grupo de estudio, mien- tras que en el grupo control se halló solamente en el $50 \%$ después de 16 semanas de cicatrización (Tabla 1). En cuanto a la continuidad de defecto hueso normal, se pudo observar histológicamente que existe una continuidad defecto hueso completa en el 80\% del grupo de estudio en comparación con solo el $30 \%$ en el grupo control (Tabla 2), determinándose así que sí existe diferencias estadísticamente significativas entre ambas muestras.

En cuanto a los hallazgos clínicos entre el grupo control y de estudio, se pudo observar que el $80 \%$ de la muestra presentó tejido óseo llenando el defecto mientras que un $30 \%$ se presentó en el grupo control, determinándose así mediante la prueba chi cuadrado que sí existe diferencia estadísticamente significativa entre ambos grupos.

\section{Efecto histológico}

Tabla 1. Comparación histológica: según grado de madurez ósea

\begin{tabular}{|c|c|c|c|c|c|c|c|}
\hline & \multicolumn{2}{|c|}{$\begin{array}{c}\text { Grupo } \\
\text { Membrana }\end{array}$} & \multicolumn{2}{|c|}{$\begin{array}{c}\text { Grupo } \\
\text { Hueso y membrana }\end{array}$} & \multicolumn{2}{|c|}{ Total } & \multirow[t]{2}{*}{$\begin{array}{l}P^{*} \\
0,036\end{array}$} \\
\hline & $\mathrm{n}$ & $\%$ & $\mathrm{n}$ & $\%$ & $\mathrm{n}$ & $\%$ & \\
\hline Presencia de células indiferenciadas & 0 & $0 \%$ & 0 & $0 \%$ & 0 & $0 \%$ & \\
\hline Proliferación y diferenciación de células indiferenciadas & 1 & $10 \%$ & 0 & $0 \%$ & 1 & $5 \%$ & \\
\hline Presencia de islotes de hueso inmaduro & 0 & $0 \%$ & 0 & $0 \%$ & 0 & $0 \%$ & \\
\hline Espículas óseas uniendo los islotes de hueso inmaduro & 4 & $40 \%$ & 0 & $0 \%$ & 4 & $20 \%$ & \\
\hline Hueso maduro compacto & 5 & $50 \%$ & 10 & $100 \%$ & 15 & $75 \%$ & \\
\hline Total & 10 & $100 \%$ & 10 & $100 \%$ & 20 & $100 \%$ & \\
\hline
\end{tabular}

Tabla 2. Comparación histológica: continuidad defecto - hueso

\begin{tabular}{|c|c|c|c|c|c|c|}
\hline & \multicolumn{2}{|c|}{$\begin{array}{c}\text { Grupo } \\
\text { Membrana }\end{array}$} & \multicolumn{2}{|c|}{$\begin{array}{c}\text { Grupo } \\
\text { Hueso y Membrana }\end{array}$} & \multicolumn{2}{|c|}{ Total } \\
\hline & $\mathrm{n}$ & $\%$ & $\mathrm{n}$ & $\%$ & $\mathrm{n}$ & $\%$ \\
\hline Ausencia de formación ósea en el reborde del defecto & 0 & $0 \%$ & 0 & $0 \%$ & 0 & $0 \%$ \\
\hline Escasa formación ósea & 2 & $20 \%$ & 0 & $0 \%$ & 2 & $10 \%$ \\
\hline Moderada formación ósea ( $2 / 3$ del defecto sin relleno) & 2 & $20 \%$ & 0 & $0 \%$ & 2 & $10 \%$ \\
\hline Elevada formación osea ( $1 / 3$ del defecto sin relleno) & 1 & $10 \%$ & 0 & $0 \%$ & 1 & $5 \%$ \\
\hline Relleno casi total del defecto (Mayor $2 / 3$ ) & 2 & $20 \%$ & 2 & $20 \%$ & 4 & $20 \%$ \\
\hline Continuidad del defecto con el hueso normal $100 \%$ & 3 & $30 \%$ & 8 & $80 \%$ & 11 & $55 \%$ \\
\hline Total & 10 & $100 \%$ & 10 & $100 \%$ & 20 & $100 \%$ \\
\hline
\end{tabular}

\section{Efecto clínico}

Tabla 3. Comparación clínica

\begin{tabular}{|c|c|c|c|c|c|c|c|c|c|}
\hline & \multicolumn{4}{|c|}{ Grupo Membrana } & \multicolumn{4}{|c|}{$\begin{array}{l}\text { Grupo } \\
\text { Hueso y Membrana }\end{array}$} & \multirow[t]{3}{*}{$\mathrm{p}^{*}$} \\
\hline & \multicolumn{2}{|c|}{ Presencia } & \multicolumn{2}{|c|}{ Ausencia } & \multicolumn{2}{|c|}{ Presencia } & \multicolumn{2}{|c|}{ Ausencia } & \\
\hline & $\mathrm{n}$ & $\%$ & $\mathrm{n}$ & $\%$ & $\mathrm{n}$ & $\%$ & $\mathrm{n}$ & $\%$ & \\
\hline Reacción inflamatoria a nivel de tejidos blandos & 5 & $50 \%$ & 5 & $50 \%$ & 2 & $20 \%$ & 8 & $80 \%$ & 0.160 \\
\hline Infecciones o exudados & 0 & $0 \%$ & 10 & $100 \%$ & 0 & $0 \%$ & 10 & $100 \%$ & \\
\hline Fracturas de la zona de trabajo & 1 & $10 \%$ & 9 & $90 \%$ & 0 & $0 \%$ & 10 & $100 \%$ & 0.305 \\
\hline Tejido blando llenando el defecto & 1 & $10 \%$ & 9 & $90 \%$ & 1 & $10 \%$ & 9 & $90 \%$ & 1 \\
\hline Tejido óseo llenando el defecto & 3 & $30 \%$ & 7 & $70 \%$ & 8 & $80 \%$ & 2 & $20 \%$ & 0.025 \\
\hline
\end{tabular}

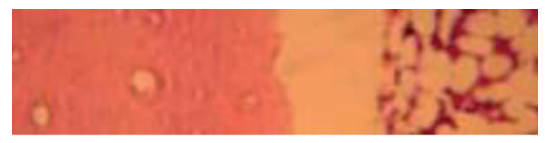

Fig 1. (H-E 40X). Imagen histológica de la tibia de conejo

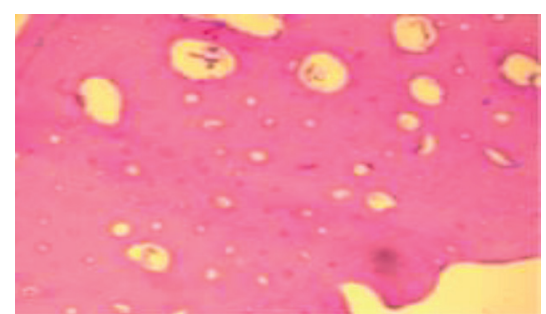

Fig 2. (H-E 200X). Imagen histológica del grupo de estudio mostrándose espacios medulares y hueso maduro compacto

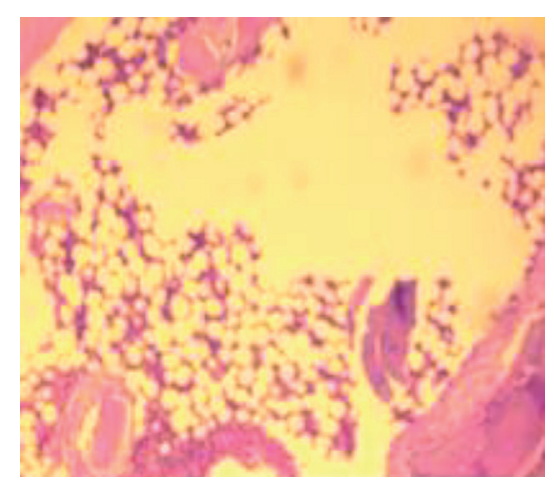

Fig 3. (H-E 40X). Imagen histológica del grupo control, en la cual se aprecia médula ósea roja y amarilla conjuntamente de islotes aislados de hueso inmaduro

\section{Discusión}

La regeneración ósea guiada se basa en la formación de nuevo hueso para el relleno de los defectos óseos; comprende el uso de membranas con funciones de barrera, las cuales deberán ser aptas para evitar la infiltración de componentes celulares (células epiteliales y conjuntivas) hacia las células osteopromotoras ${ }^{7}$, 8 .

En este estudio experimental utilizamos la membrana amniótica liofilizada como barrera biológica en combinación con el injerto óseo humano liofilizado en este proceso de regeneración ósea, 
en el cual se crearon defectos en el tejido óseo de la región más inferior de las extremidades posteriores de los conejos (tibia).

Se recomienda un período de cicatrización de 8 semanas a más, con un tamaño de $4 \mathrm{~mm}$ a más de los defectos óseos, para la evaluación de la reabsorción de los materiales y la cantidad de regeneración ósea en las tibias de los conejos.

Se determinó el efecto de esta combinación de membrana amniótica y hueso humano liofilizado, realizando la valoración en el proceso de regeneración ósea guiada en dos niveles: a nivel clínico (realizando la valoración macroscópica de la zona donde se colocó la membrana después de 16 semanas de cicatrización) y a nivel histológico (mediante las observaciones microscópicas de las muestras obtenidas del proceso de regeneración ósea después de 16 semanas de cicatrización, para lo cual se tomaron en cuenta 2 criterios: la continuidad del defecto óseo - hueso normal y el grado de madurez ósea). Con este fin se obtuvieron en total 20 muestras (10 del grupo de estudio y 10 del grupo control), las cuales fueron analizadas clínica e histológicamente.

En referencia a trabajos anteriores aplicando los injertos óseos en combinación con membrana amniótica como barrera en el proceso de regeneración ósea, estos hasta la actualidad no existen o todavía no han sido dados a conocer a la comunidad científica. La mayoría de trabajos en los cuales se utiliza esta membrana amniótica liofilizada se puede encontrar en el mundo de la oftalmología ${ }^{8,9,10,11,12}$ y a sea que estén siendo utilizados en intervenciones quirúrgicas de reconstrucciones de la superficie ocular $^{10,11}$ o en casos de intervenciones quirúrgicas de Pterigon ocular 16, en los cuales se utiliza esta membrana como una barrera para evitar el paso de microorganismos y facilitar la reepitealización de la zona de trabajo. En todos los casos intervenidos, el uso de la membrana amniótica liofilizada se mostró como una técnica segura, ya que en ningún caso se encontraron complicaciones intra o post-operatorias, ni se observó un empeoramiento de la zona del trabajo. Así mismo los resultados obtenidos por los estudios se mostraron estables por un período amplio de seguimiento. Esto se puede explicar gracias a que esta membrana amniótica proporciona una membrana basal, la cual favorece la migración y la diferenciación celular ${ }^{13,14}$. En este estudio podemos decir que favoreció la migración de células multipotenciales que posteriormente se diferenciaron en osteoblastos, reduce la inflamación de la zona por debajo de la membrana ${ }^{13}$ y actúa como sello biológico evitando el pase de diversas clases de microorganismos hacia la herida; en este estudio evita que diversos tipos de tejidos, especialmente el epitelial y el conjuntivo, ingresen dentro del defecto óseo, impidiendo de esta manera una regeneración completa.

Existen 2 trabajos reportados en odontología que nos servirán como referencia y para comparar los resultados que hemos obtenido.

Yabar y col. ${ }^{6}$ en el año 2010 evaluaron el efecto de la membrana amniótica en el proceso de regeneración ósea guiada, tanto clínica e histológicamente. Ellos encontraron clínicamente que el $80 \%$ de la muestra presentaba tejido duro llenando el defecto, en comparación con nuestros resultados que hueso humano liofilizado en combinación con membrana amniótica se logró obtener $100 \%$ de la muestra con tejido óseo llenando el defecto. Histológicamente, utilizando el índice de Helpe, encontraron un $87,5 \%$ de hueso compacto maduro mientras nosotros encontramos $100 \%$.

Rios y col, ${ }^{15}$ en el ańo 2011, realizaron un estudio en el cual compararon la densidad ósea en defectos óseos en tibias de conejos tratados con membrana amniótica liofilizada y membrana de colágeno, que al ser evaluados a las 5 semanas de cicatrización, según tomografía Cone Beam, mostraron niveles similares de densidad ósea con ambas membranas. En nuestro caso, utilizamos esta membrana amniótica en combinación con hueso humano liofilizado e irradiado con radiación gamma a 25 Kgray y obtuvimos tanto clínica como histológicamente resultados significativamente diferentes con el solo uso de membrana amniótica.

\section{Conclusiones}

- Clínicamente, el empleo de la membrana amniótica humana liofilizada en combinación con hueso liofilizado, produjo menor frecuencia de reacción inflamatoria que el grupo que solo utilizó membrana amniótica.

- Histológicamente, el empleo de membrana amniótica humana liofilizada en combinación con hueso liofilizado produjo una mayor frecuencia de presentación de hueso maduro compacto y continuidad con el hueso normal que el grupo que solo utilizó membrana amniótica.

- El uso de membrana amniótica humana liofilizada en combinación con hueso liofilizado en defectos óseos en tibias de conejos, después de 16 semanas, produjo una mayor regeneración ósea guiada que cuando se usó solo la membrana amniótica como barrera.

\section{Referencias bibliográficas}

1. Peral Cagigal B, Redondo González Lm, Verrier Hernández A, Serrat Soto A, Torres Nieto Ma, Vaquero Puerta C. Estudio experimental sobre la regeneración ósea mandibular de la rata con diferentes biomateriales. Rev Esp Cirug Oral y Max 2008; 30(1): 15 - 24

2. Hiatt WH, Schallhorn RG, Aaronian AJ. The induction of new bone and cementum formation. IV. Microscopic examination of the periodontium following human bone and marrow allograft, autograft and non-graft periodontal regenerative procedures. J of Periodont 1978; 49:495-512

3. Keith J.. Localized Ridge Augmentation With a Block Allograft Followed by Secondary Implant Placement: A Case Report. Int J Periodont Rest Dent. 2004; 24:11-17.

4. Zermeño Ibarra J, Cepeda Bravo J. Comparación entre membranas biodegradables y no degradables en la terapia de regeneración tisular guiada, Revista ADM 1999; 56(1): 39-43.

5. Nasr HF, Aichelmann-Reidy ME, Yukna RA. Bone and bone substitutes. Periodontology 2000 1999;19:74-86

6. Yabar. J. Efecto de la membrana amniótica liofilizada como barrera en el proceso de regeneración ósea en tibia de conejo. Tesis para obtener el titulo d cirujano dentista UNMSM 2010.

7. Glowaki J. Tissue response to bone derived implants. In: Habal MB, Reddi HA. Bone grafts \& bone substitutes 1 Ed. W. B. Philadelphia: Saunders Company, Harcourt Brace Jovanovich, Inc; 1994. p. 84-92.

8. Eill Sr, Cobb C, Rapley J, Glaros A, Spencer P., In vivo comparison 
of synthetic osseous graft materials. A preliminary study. J Clin Periodontol 1999; 26: 239-245.

9. Robert H. Lyford, Michael P. Mills, MS Charles I. Knapp,E. Todd Scheyer, James T. Mellonig, Clinical Evaluation of FreezeDried Block Allografts for Alveolar Ridge Augmentation: A Case Series. (Int J Periodontics Restorative Dent 2003;23:417-425

10. Walker T, Johnny B. Localized Ridge Augmentation Using a Block Allograft with Subsequent Implant Placement: A Case Series. Int J Periodontics Restorative Dent 2008;28:509-515.
11. Nikolaos D et al. Long-term stability of autogenous bone grafts following combined application with guided bone regeneration. Clin. Oral Impl. Res. 2005; 16: 133-139

12. Stangogiannis-druya, Martínez M, Paz 1., Márquez K. Trasplante de membrana amniótica o Auto injerto conjuntival en Pterigión Nasal Recidivantes. Rev. Oftalmol. Venez. 2004 v.60 n.4.

13. La Rosa Garza M De, Cepeda J. Regeneración ósea guiada de cara al ańo 2000, consideraciones clínicas y biológica. Revista ADM 2000. 57(4): 147-153.
14. Leonardo j. De luca (Laboratorios Burnet).Aborto bovino. Boletín informativo; 2008. p. 12-18.

15. Rios K, Meza E, Huamani J. Membrana Amniotica liofilizada y regeneración osea. Estudio Tomografico e Histomorfometrico. Tesis para obtener el grado de Magister UPCH 2012.

\section{Conflicto de intereses}

El autor declara que no existió conflicto de intereses en relación con la publicación de este documento.

Este estudio fue financiado únicamente por el autor de este artículo. 